\section{FRI0686 NEGATIVE ASSOCIATIONS FOR FASTING BLOOD GLUCOSE, CHOLESTEROL AND TRIGLYCERIDE LEVELS WITH THE DEVELOPMENT OF GIANT CELL ARTERITIS}

K. Jakobsson ${ }^{1,2}$, L.T. Jacobsson ${ }^{1,3}$, A.J. Mohammad ${ }^{4,5}$, K.J. Warrington ${ }^{6}$, E.L. Matteson ${ }^{6}$, C. Turesson ${ }^{1,2}$. ${ }^{1}$ Department of Clinical Sciences, Malmö, Lunds University; ${ }^{2}$ Department of Rheumatology, Skåne University Hospital, Malmö; ${ }^{3}$ Department of Rheumatology \& Inflammation Research, The Sahlgrenska Academy, University of Gothenburg, Institute of Medicine, Gothenburg; ${ }^{4}$ Department of Rheumatology, Skåne University Hospital, Lund, Sweden; ${ }^{5}$ Vasculitis and Lupus Clinic, Addenbrooke's Hospital, Cambridge, United Kingdom; ${ }^{6}$ Division of Rheumatology, Mayo Clinic College of Medicine and Science, Rochester, Minnesota, United States

Background: Giant cell arteritis (GCA) is the most common vasculitis among patients age $>50$ years in north European countries. Although ethnic factors and age clearly play a role, the etiology of the disease is largely unknown. Studies of predictors are therefore of major interest. A meta-analysis of observational studies demonstrated that patients with GCA have a significantly reduced prevalence of diabetes at the time of diagnosis (1).

Objectives: To investigate metabolic features prior to diagnosis of GCA in a nested case-control study.

Methods: Individuals who developed GCA after inclusion in a population-based health survey (the Malmö Preventive Medicine Project; $\mathrm{N}=33346$ ) were identified by linking the health survey database to the local patient administrative register and the national patient register. A structured review of medical records was performed. Four controls for every validated case, matched for sex, year of birth and year of screening, were selected from the database. Fasting blood samples had been obtained and analyzed using standard methods as part of the health survey. Potential predictors of GCA were examined in conditional logistic regression models.

Results: There were 76 cases with a confirmed clinical diagnosis of GCA $(61 \%$ female; $65 \%$ biopsy positive; $95 \%$ fulfilled the ACR criteria for GCA). The mean age at diagnosis was 70 years, and the median time from screening to diagnosis was 20 years (range $2-32$ ). The cases had significantly lower fasting blood glucose (fB-glucose) at baseline screening compared to controls [mean 4.7 vs 5.1 $\mathrm{mmol} / \mathrm{l}$, odds ratio (OR) 0.49 per mmol//; $95 \%$ confidence interval (Cl) $0.30-0.79$ ]. Current smokers had a reduced risk of GCA (OR $0.35 ; 95 \% \mathrm{Cl} 0.18-0.70)$. The negative association between baseline fB-glucose and GCA remained significant in analysis adjusted for smoking (OR $0.46 \mathrm{per} \mathrm{mmol/l;} 95 \% \mathrm{Cl} 0.28-0.76$ ). Both cholesterol (mean 5.6 vs $6.0 \mathrm{mmol} / \mathrm{l}$ ) and triglyceride levels (median 1.0 vs $1.2 \mathrm{mmo} / \mathrm{l}$ ) were lower among the cases at baseline screening, with significant negative associations with subsequent GCA in crude and smoking-adjusted (ORs with $95 \%$ Cls 0.66 per mmol//; $0.46-0.94$ for cholesterol, 0.33 per mmol//; $0.16-0.69$ for triglycerides) models. The effect of fB-glucose on the risk of GCA was stronger in men compared to women (smoking-adjusted ORs per mmol/l $0.11 ; 95 \% \mathrm{Cl} 0.03-0.37$, and $0.77 ; 95 \% \mathrm{Cl} 0.50-1.18$, respectively). Apart from this, results were similar in women and men.

Conclusions: Development of GCA was associated with lower fB-glucose, lower cholesterol and triglyceride levels at baseline, all adjusted for current smoking. These findings are in line with the previous findings of a reduced prevalence of diabetes mellitus at the time of diagnosis of GCA (1). This suggests that metabolic factors influence the development of GCA.

References:

[1] Ungprasert P et al Mod Rheumatol. 2016;26(3):410-414.

Disclosure of Interest: None declared

DOI: 10.1136/annrheumdis-2017-eular.4245

\section{FRI0687 BODY MASS INDEX, SMOKING, SOCIOECONOMIC STATUS AND THE RISK OF GIANT CELL ARTERITIS}

K. Jakobsson ${ }^{1,2}$, L.T. Jacobsson ${ }^{1,3}$, A.J. Mohammad ${ }^{4,5}$, K.J. Warrington ${ }^{6}$, E.L. Matteson ${ }^{6}$, C. Turesson ${ }^{1,2}{ }^{1}$ Department of Clinical Sciences, Lunds University; ${ }^{2}$ Department of Rheumatology, Skåne University Hospital, Malmö; ${ }^{3}$ Department of Rheumatology \& Inflammation Research, The Sahlgrenska Academy, University of Gothenburg, Institute of Medicine, Gothenburg; ${ }^{4}$ Department of Rheumatology, Skåne University Hospital, Lund, Sweden ${ }^{5}$ Vasculitis and Lupus Clinic, Addenbrooke's Hospital, Cambridge, United Kingdom; ${ }^{6}$ Division of Rheumatology, Mayo Clinic College of Medicine and Science, Rochester, Minnesota, United States

Background: Few studies have examined predictors of giant cell arteritis (GCA). A negative association between body mass index (BMI) and development of GCA has been reported $(1,2)$. There is limited information on the impact of smoking and socioeconomic factors on the risk of GCA.

Objectives: To further investigate the relation between BMI and GCA, and also assess the role of smoking and socioeconomic factors in this context.

Methods: Two population based health-surveys, The Malmö Preventive Medicine Program (MPMP) and the Malmö Diet Cancer Study (MDCS), performed in the same catchment area between 1974 and 1996. In the MPMP, 33346 subjects (33\% women), and in the MDCS, 30447 subjects (60\% women) were included. Both surveys included standard physical examinations and self-administered questionnaires. Subjects were classified as blue-collar workers or white-collar workers, using the Socioeconomic Index (SEI) based on self-reported job titles in the Swedish national censuses.
Individuals who developed GCA after inclusion in the two health surveys were identified by linking the health survey databases to local and national patient registers. A structured review of the medical records was performed. Four controls for every validated case, matched for sex, year of birth and year of screening, were selected from the corresponding databases. Potential predictors of GCA were examined in conditional logistic regression models. This is an extension of a previous study, adding more incident cases (2).

Results: A total of 138 cases with a confirmed clinical diagnosis of GCA (median age at diagnosis 71 years; $72 \%$ female; $66 \%$ biopsy-positive; $94 \%$ fulfilled the ACR criteria for GCA) were included. The median time from screening to diagnosis was 15 years (range $0-32$ ).

The cases who subsequently developed GCA had significantly lower BMI at baseline (24.3 vs $25.3 \mathrm{~kg} / \mathrm{m}^{2}$, odds ratio (OR) 0.91 per $\mathrm{kg} / \mathrm{m}^{2} ; 95 \%$ confidence interval (Cl) $0.86-0.97$ ) and were less likely to be current smokers when entering the health survey (OR $0.56 ; 95 \% \mathrm{Cl} 0.33-0.94$ ). There was no difference in the proportion with low level of formal education between cases and controls (OR $1.27 ; 95 \% \mathrm{Cl} 0.66-2.44)$. Blue-collar workers tended to be less likely to develop GCA than white-collar workers (OR $0.53 ; 95 \% \mathrm{Cl} 0.28-1.00)$. This association reached statistical significance in women (OR $0.32 ; 95 \% \mathrm{Cl} 0.13-0.81$ ) but not in men (OR $0.87 ; 95 \% \mathrm{Cl} 0.35-2.16)$. In multivariate analysis, including both variables and the SEl, the impact of $\mathrm{BMI}(\mathrm{OR} 0.85 ; 95 \% \mathrm{Cl} 0.74-0.96)$ and smoking (OR 0.26; 95\% $\mathrm{Cl} 0.12-0.60$ ) remained significant.

Conclusions: In this study, the negative association between $\mathrm{BMI}$ and subsequent GCA was confirmed, and there was an independent protective effect of smoking. Socioeconomic status, reflected by occupation later in life rather than level of formal education, may also influence the risk of developing GCA.

\section{References:}

[1] Larsson K et al Ann Rheum Dis 2006; 65:529-532.

[2] Jakobsson $\mathrm{K}$ et al Rheumatology 2015; 54:433-440.

Disclosure of Interest: None declared

DOI: 10.1136/annrheumdis-2017-eular.4021

\section{FRI0688 PRERA (PREDICTING RHEUMATOID ARTHRITIS): PRELIMINARY FINDINGS FROM AN ON-GOING PROSPECTIVE STUDY OF SEROPOSITIVE AND SERONEGATIVE INDIVIDUALS AND THEIR RISK FOR DEVELOPING RA}

M. Mayr ${ }^{1}$, M. Hucke ${ }^{1}$, V. Nell-Duxneuer ${ }^{1}$, T. Stamm ${ }^{1}$, G. Steiner ${ }^{1}$,

T. Perkmann ${ }^{1}$, E. Hitzelhammer ${ }^{2}$, J. Smolen ${ }^{1}$, K. Machold ${ }^{1}$, H. Radner ${ }^{1}$.

${ }^{1}$ Rheumatology, Medical University of Vienna; ${ }^{2}$ Health Centers of The Vienna General Health Insurance, Wien, Austria

Background: A characteristic feature of RA is the presence of autoantibodies $(A A B)$, such as rheumatoid factor (RF) and antibodies against citrullinated peptides (ACPA). RF and ACPA are not only diagnostically helpful but are also prognostic indicators for progression of joint destruction.

Objectives: This is an on-going prospective study to investigate the prevalence of $A A B$ in healthy individuals presenting to community health centres and to subsequently determine the incidence of RA in AAB-positive individuals compared to $A A B$-negative persons over five years considering the presence of certain risk factors.

Methods: Blood drawn at the time of the screening examination was anonymously analyzed for the presence of $A A B$. $A A B$ positive and age and sex matched $A A B$ negative individuals (2 for each $A A B$ positive subject) were enrolled for further assessment at the central outpatient clinic and examined every 6 months over 5 years. Assessment included laboratory testing (AAB, acute phase parameters), questionnaires on nutrition, lifestyle and general health, 28 joint counts (SJC28, TJC28), the health assessment questionnaire ( $\mathrm{HAQ}$ ), and visual analogue scales for pain and global health. Risk factors were analysed by clusters according to EULAR recommendations for terminology of individuals at risk of RA: hereditary, environmental, systemic autoimmunity and unclassified arthritis and the number of clusters per individual was reported at baseline (1). The primary outcome was the development of RA; individuals lost to follow up were telephone-interviewed for signs and symptoms of RA.

Results: 4858 sera were obtained from which $148(3 \%)$ were seropositive (14 ACPA, 124 RF, 10 both). 113 individuals $(2,3 \%)$ were followed over 5 years. 37 $(32,7 \%)$ were seropositive of whom $32(28,3 \%)$ were RF-positive, $2(5,4 \%)$ were ACPA-positive and $3(8,1 \%)$ positive for both. We found no significant differences in demographics and risk factors between seropositive and seronegative individuals at baseline (Table 1). The number of risk clusters per individuals is reported in

\begin{tabular}{llcc}
\hline Risk Factors & & Seronegative $(n=76)$ & Seropositive $(n=37)$ \\
\hline Age & & $54,8( \pm 12,3)$ & $57,6( \pm 10,3)$ \\
Genetic & Sex (\% female) & $50(66 \%)$ & $23(62 \%)$ \\
& Family history & $11(14 \%)$ & $3(8 \%)$ \\
Environmental & Smoking & $20(26 \%)$ & $8(22 \%)$ \\
& BMI $>25 \mathrm{~kg} / \mathrm{m}^{2}$ & $37(49 \%)$ & $14(38 \%)$ \\
\multirow{4}{*}{ Unclassified Arthritis } & $20(29 \%)$ & $16(49 \%)$ \\
& Low education & $0(0-2)$ & $0(0-2)$ \\
& SJC28 & $0(0-2)$ & $0(0-3)$ \\
& TJC28 & $8,9( \pm 16,8)$ & $5,1( \pm 10,7)$ \\
& Patient Global & $0,07( \pm 0,17)$ & $0,05( \pm 0,15)$ \\
& HAQ & $0,29( \pm 0,46)$ & $0,21( \pm 0,27)$ \\
\hline
\end{tabular}




\begin{tabular}{|c|c|c|c|c|c|}
\hline \multirow{2}{*}{$\begin{array}{l}\text { Number of } \\
\text { risk clusters } \\
\text { per } \\
\text { individuals }\end{array}$} & \multirow{2}{*}{$\begin{array}{l}\text { Number of } \\
\text { Individuals (59) }\end{array}$} & \multicolumn{4}{|c|}{ RISK CLUSTER } \\
\hline & & $\begin{array}{c}\text { Systemic } \\
\text { autoimmunity }\end{array}$ & $\begin{array}{c}\text { Environmental } \\
\text { risk factors }\end{array}$ & $\begin{array}{c}\text { Genetic risk } \\
\text { factors }\end{array}$ & $\begin{array}{c}\text { Unclassifiled } \\
\text { Arthritis }\end{array}$ \\
\hline NONE & $8(7.15)$ & & & & \\
\hline \multirow{4}{*}{ ONE } & $19(16.858)$ & & & & \\
\hline & $10(8.85)$ & & & & \\
\hline & $2(1.85)$ & & & & \\
\hline & 0 & & & & \\
\hline \multirow{6}{*}{ Two } & 35 (31\%) & & & & \\
\hline & $9(8 \% 6)$ & & & & \\
\hline & $5(4,48)$ & & & & \\
\hline & $2(1.85)$ & & & & \\
\hline & $\circ$ & & & & \\
\hline & 0 & & & & \\
\hline \multirow{4}{*}{ THREE } & $17(155)$ & & & & \\
\hline & $3(2.78)$ & & & & \\
\hline & 0 & & & & \\
\hline & 0 & & & & \\
\hline FOUR & $2(2.88)$ & & & & \\
\hline
\end{tabular}

figure 1. Until now, 43 people were followed for 5 years; additional 52 telephone interviews were conducted. No evidence of RA (clinically or by history) was found. Conclusions: By now none of the followed individuals had any evidence of inflammatory joint disease based on patient-telephone interviews conducted and completed 5-year follow-up examinations. We were unable to find evidence for practical value of routine $A A B$ screening in healthy individuals without clinical signs of inflammatory joint diseases.

References:

[1] Gerlag et al. (2012) Ann Rheum Dis 2012;71:638-641.

Disclosure of Interest: None declared

DOI: 10.1136/annrheumdis-2017-eular.5951

\section{FRI0689 LIPID PEROXIDATION AS RISK FACTOR FOR ENDOTHELIAL DYSFUNCTION IN ANTIPHOSPHOLIPID SYNDROME (APS) PATIENTS}

L. Stojanovich, N. Stanisavljevic, A. Djokovic, M. Zdravkovic. Internal medicine department, University Hospital Center Bezanijska Kosa, Belgrade, Serbia

Background: APS pathophysiology is not clear enough yet since it has been implicated that aPL can activate cells (endothelial cells, monocytes, platelets), interfere with hemostatic reactions and activate complement reactions [1,2]

Objectives: The aim of this study was to evaluate oxidative stress markers and it relations to endothelial damage as risk factor for thrombosis in patients with primary (PAPS) and secondary (SAPS) antiphospholipid syndrome (APS) in correlation to traditional risk factors.

Methods: Flow mediated (FMD) and nitroglycerine (NMD) induced dilation of the brachial artery were studied in 140 APS patients (90 PAPS, 50 SAPS) and 40 controls matched by age, sex and conventional risk factors for atherosclerosis. Markers of oxidative stress: lipid hydroperoxydes ( $\mathrm{LOOH}$ ), advanced oxidation protein products (AOPP), total sulfhydryl grups (tSHG) and paraoxonase 1 activity (PON1) were determined by spectrophotometric method.

Results: Oxidative stress dominate in APS patients. $\mathrm{LOOH}$ and AOPP correlate to lipid fractions $(p<0.05)$, unlike PON1, tSHG that correlated to antiphospholipid antibody positivity $(p<0.05)$. FMD was lower in APS patients comparing to controls $(p<0.001)$. Cholesterol is independent variable for FMD impairment in control group ( $p=0.011) ; \mathrm{LOOH}$ in PAPS $(\mathrm{p}=0.004) ; \mathrm{LOOH}, \mathrm{aCL}$ and triglycerides in SAPS patients $(p=0.009, p=0.049$ and $p=0.012$, respectively).Combined predictive of $\mathrm{aCL}$ and $\mathrm{LOOH}$ is better for FMD impairment than $\mathrm{LOOH}$ alone in both PAPS and SAPS patients (AUC $0.727, \mathrm{p}=0.001,95 \% \mathrm{Cl} 0.616-0.837$ and AUC 0.824 , $\mathrm{p}<0.001,95 \% \mathrm{Cl}$ 0.690-0.957, repectively).

Conclusions: Endothelial dysfunction is doubtlessly present in APS patients with oxidative imbalance as additional risk factor among other risk factors for clinical event. Anticardiolipin antibodies affect endothelial dependent vasodilatation in SAPS patients. We demonstrated synergistic effect of aCL and $\mathrm{LOOH}$ as risk for endothelial impairment in both PAPS and SAPS patients.

\section{References:}

[1] Foltyn Zadura A, Memon AA, Stojanovich L, Perricone C, Conti F, Valesini G et al (2015) Factor H Autoantibodies in Patients with Antiphospholipid Syndrome and Thrombosis. J Rheumatol 42(10):1786-93.

[2] Stalc M, Poredos P, Peternel P, Tomsic M, Sebestjen M, Kveder T (2006) Endothelial function is impaired in patients with primary antiphospholipid syndrome. Thromb Res 118:455-461.

[3] Becarevic M, Stojanovich L, Ignjatovic S, Dopsaj V. The IgM isotype of antiannexin A5 antibodies and multiple positivity of conventional antiphospholipid antibodies: increasing the number of clinical manifestations of primary antiphospholipid syndrome. Clinical Rheumatology 03/2016; doi: 10.1007/ s10067-016-3230-0.
[4] Ames PR, Batuca JR, Ciampa A, lannaccone L, Delgado Alves J (2010) Clinical relevance of nitric oxide metabolites and nitrative stress in thrombotic primary antiphospholipid syndrome. J Rheumatol 37:2523-2530.

Acknowledgements: This work was supported by research grant number 175041, and TR 32040 for 2011 - 2017, issued by the Ministry of Science of the Republic of Serbia.

Disclosure of Interest: None declared

DOI: 10.1136/annrheumdis-2017-eular.2872

\section{FRI0690 RISK OF FRACTURE AMONG PATIENTS WITH GOUT: A POPULATION-BASED COHORT STUDY}

A. Abdul Sultan ${ }^{1}$, R. Whittle ${ }^{1}$, S. Muller ${ }^{1}$, E. Roddy ${ }^{1,2}$, C. Mallen $^{1}$, M. Blagojevic-Bucknall ${ }^{1}$, T. Helliwell ${ }^{1}$, S. Hider ${ }^{1,2}$, Z. Paskins $^{1,2},{ }^{1}$ Research Institute for Primary Care \& Health Sciences, Keele University, Staffordshire; ${ }^{2}$ Haywood Academic Rheumatology Centre, Staffordshire and Stoke-on-Trent Partnership Trust, Stoke-on-Trent, United Kingdom

Background: Gout is the most common type of inflammatory arthritis, affecting $2.4 \%$ of adults in the UK and is associated with a number of co-morbidities. Our understanding of the association between gout and fracture risk is limited with previous studies offering conflicting results.

Objectives: To determine the risk of fracture among gout patients and assess the potential impact of urate-lowering therapy (ULT) on fracture risk.

Methods: Utilising primary care records from Clinical Practice Research Datalink we identified patients with gout between 1990 and 2004 who were followed up until 2015. Each gout patient was individually matched to 5 individuals without gout based on age, sex, and registered practice. Absolute rate (AR) of fracture and hazard ratios (HR) were calculated using Cox regression models. We further stratified our analysis by age, gender and ULT prescription.

Results: We matched 35,857 patients with incident gout to 148,407 controls. Overall, we found no increased risk of fracture among gout patients compared to controls. However, men with no evidence of ULT had higher absolute risk of fracture compared to controls ( $A R=39$ versus 26 per 10,000 person-years) corresponding to a $23 \%(\mathrm{HR}=1.23 ; 95 \% \mathrm{Cl} 1.12-1.36)$ increased risk. The risk was particularly high for vertebral $(\mathrm{HR}=1.50 ; 95 \% \mathrm{Cl} 1.20-1.87)$ and wrist fracture $(\mathrm{HR}=1.45 ; 95 \% \mathrm{Cl} 1.21-1.74)$. Those treated with ULT had a 12\% (HR=0.88; $95 \% \mathrm{Cl}$ 0.79-0.98) lower risk of fracture. Similar findings were not observed for women.

Conclusions: We found higher risk of vertebral and wrist fractures among men with gout not prescribed ULT. Those prescribed ULT had lower risk of fracture compared to the general population. Further research is needed to understand the role of ULT in fracture prevention.

Acknowledgements: CDM is funded by the National Institute for Health Research (NIHR) Collaborations for Leadership in Applied Health Research and Care West Midlands, the NIHR School for Primary Care Research and a NIHR Research Professorship in General Practice. TH is funded by a NIHR Clinical Lectureship in General Practice and AAS is funded by NIHR Postdoctoral Fellowship. The views expressed are those of the author(s) and not necessarily those of the NHS, the NIHR or the Department of Health

Disclosure of Interest: None declared

DOI: 10.1136/annrheumdis-2017-eular.3761

\section{FRI0691 ASSOCIATION BETWEEN PERIODONTITIS AND THE RISK OF PALINDROMIC RHEUMATISM: A NATIONWIDE, POPULATION-BASED, CASE-CONTROL STUDY}

H.-H. Chen ${ }^{1}$, W.-C. Chao ${ }^{1}$, Y.-M. Chen ${ }^{1}$, D.-Y. Chen ${ }^{2} .{ }^{1}$ Department of Medical Research; ${ }^{2}$ Department of Internal Medicine, Taichung Veterans General Hospital, Taichung, Taiwan, Province of China

Background: Some patients with palindromic rheumatism (PR) may develop a chronic connective tissue disease, mainly rheumatoid arthritis (RA). About one to two-thirds of PR patients developed RA during a period of follow-up. Periodontitis (PD) has been found to be associated with RA risk. However, the association between PD and PR risk is unknown.

Objectives: To estimate the association between a history of PD and the risk of incident PR.

Methods: This study used a nationwide, administrative database to identify PR cases and non-PR controls. After exclusion of individuals with rheumatoid arthritis, systemic lupus erythematosus, Sjögren's syndrome, systemic sclerosis, dermatomyositis or polymyositis before the first PR diagnosis date (index date), we identified 4,421 newly-diagnosed PR cases from 2007 to 2012 and randomly selected 44,210 non-PR controls matched (1:10) for sex, age, and the year of the index date. After adjusting for comorbid diabetes mellitus, we estimated odds ratios (ORs) with $95 \%$ confidence intervals (Cls) by conditional logistic regression analysis to quantify the association between a history of PD and the risk of PR. The influences of the lag time and severity of PD were examined by calculating ORs for subgroups of patients based on time interval between the last PD-related visit and the index date and PD-related cumulative cost and visit number.

Results: This study showed an association between a history of PD and newly diagnosed PR $(\mathrm{OR}, 1.51 ; 95 \% \mathrm{Cl}, 1.41-1.61)$. The association remained significant after variation of PD definitions. The magnitude of the association was 